\title{
Machine Learning para la mejora de la experiencia con MOOC: el caso de la Universitat Politècnica de València
}

\author{
Machine Learning to improve the MOOC experience: the case of the \\ Universitat Politècnica de València
}

\author{
Jorge Ángel Martínez Navarro \\ Escuela de Arte y la Escuela Superior de Diseño de Murcia (España) \\ ajorgemartinez@gmail.com \\ Ignacio Despujol Zabala \\ Universitat Politècnica de València (España) \\ ndespujol@asic.upv.es
}

Recibido: 01/02/2021

Aceptado: 06/04/2021

Publicado: 01/06/2021

\section{RESUMEN}

El trabajo que se presenta tiene como objetivo el diseño de una propuesta de mecanismos automatizados fundamentados en machine learning para la mejora de la experiencia de los participantes en los cursos MOOC de la Universitat Politécnica de Valencia y la reducción de las tasas de abandono. Siguiendo una estrategia de investigación basada en el diseño IBD, en la que se ha priorizado siempre las decisiones pedagógicas por encima de las propias analíticas de datos, se han realizado tres iteraciones con distintos patrones metodológicos (revisión sistemática de literatura, machine learning basado en los datos de 260 cursos y más de 700.000 estudiantes, y creación de mecanismos automatizados) que siempre finalizan con la presentación de resultados y la realimentación por parte del equipo de la universidad. Las principales conclusiones de este trabajo indican que, de los veinticinco indicadores pedagógicos de abandono referidos por las revisiones bibliográficas en la iteración 1, solo se validan diez de ellos con los cursos de la UPV (no se tienen datos automáticos ni automatizables de los otros), y de esos finalmente solo seis de ellos son posibles predictores del abandono del alumnado, con los datos utilizados. Se proponen finalmente un conjunto de mecanismos automatizados que se aplicarán en la plataforma EdX de la universidad, para la mejora de la experiencia de los usuarios y la reducción de la tasa de abandonos en los cursos.

\section{PALABRAS CLAVE}

MOOC, Machine Learning, Analíticas de aprendizaje, predicciones, abandono

\begin{abstract}
The aim of this paper is to design a proposal for automated mechanisms based on machine learning to improve the experience of participants in MOOC courses at the Universitat Politécnica de Valencia and reduce dropout rates. Following a desing based research DBR design, in which pedagogical decisions have always been prioritised over data analytics, three iterations have been carried out with different methodological patterns (systematic literature review, machine learning based on data from 260 courses and 700.000 students, and creation of automated mechanisms) that always end with the presentation of results and feedback from the university team. The main conclusions of this work indicate that, of the twentyfive pedagogical dropout indicators referred to by the literature reviews in iteration 1, only ten of them are validated with UPV courses (no automated or automatable data are available for the others), and of those finally only six of them are possible predictors of student dropout, with the data used. Finally, a set of automated mechanisms are proposed to be applied in the university's EdX platform to improve the user experience and reduce the dropout rate in the courses.
\end{abstract}




\section{KEYWORDS}

MOOC, Machine Learning, Learning analytics, predictions, dropout.

\section{CITA RECOMENDADA}

Martínez, J.A. \& Despujol, I. (2021). Machine Learning para la mejora de la experiencia con MOOC: el caso de la Universitat Politècnica de València. Revista Interuniversitaria de Investigación en Tecnología Educativa, 10, 91-104. https://doi.org/10.6018/riite

\section{Principales aportaciones del artículo y futuras líneas de investigación:}

- Propuesta de mecanismos automatizados de mejora de la tasa de abandono en la plataforma MOOC de una universidad a partir de la validación, mediante técnicas de machine learning, de un conjunto de indicadores de deserción obtenidos en revisiones sistemáticas de literatura.

- Como líneas futuras de investigación se propone la introducción de nuevos mecanismos que tengan como base la interacción en el foro de los estudiantes. Además, es interesante la validación de estos mecanismos propuestos en otros contextos distintos a los de los MOOC de la UPV.

\section{INTRODUCCIÓN}

Tras el éxito de las primeras experiencias que realizó la Universidad de Stanford con los MOOC (Massive Open Online Courses) en 2012, muchas universidades de todo el mundo (además de iniciativas privadas) comenzaron a experimentar con estos cursos. Cinco años después del primer MOOC, más de 700 universidades de todo el mundo habían producido 6.850 MOOC, y más de 58 millones de estudiantes se habían inscrito en esos cursos (Shah, 2016). Este es el caso Universitat Politècnica de València (UPV) que, con sus más de 230 cursos diferentes y más de 700.000 estudiantes matriculados en estas experiencias, es la institución europea con más cursos MOOC realizados (Ruipérez-Valiente, 2020).

Sin embargo, a pesar de los beneficios de la aplicación de la tecnología, son muchas las investigaciones (Veletsianos \& Shepherdson, 2016; Rolfe, 2015; Bartolomé-Pina \& Steffens, 2015) que afirman que los MOOC no son aún una alternativa de educación consolidada a la educación tradicional y que, cuestiones básicas como la deserción de los estudiantes, siguen suponiendo un problema a la hora de considerarlos una aportación clara en los modelos de educación profesionalizadora.

Dado el gran volumen de datos disponibles algunas de las soluciones a estos problemas se centran en las analíticas de datos (learning analytics); y para el aprovechamiento de esos datos, ha surgido como alternativa la técnica de Machine Learning. El término Machine Learning (en adelante ML) es una disciplina de la inteligencia artificial cuyas técnicas hacen posible el aprendizaje de los ordenadores a partir del entrenamiento con grandes volúmenes de datos (Russo et al, 2016). El objetivo es crear programas que puedan generar patrones de comportamiento potenciales a partir de unos datos (Bishop, 2006); se trata de obtener modelos efectivos que permitan predecir el comportamiento del alumnado para poder operar a partir de ellos (Mohri et al., 2012).

Este estudio nace de la necesidad de la UPV de mejorar su experiencia y la de sus estudiantes en su plataforma MOOC, y del convencimiento de que una estrategia basada en el ML podría ser de utilidad para diseñar mecanismos automatizados que incidieran de forma masiva en la mejora de la experiencia de los cursos por parte de sus participantes.

Para ello se ha realizado una investigación basada en el diseño (IBD), formada por tres fases (Figura 1) que se logran completar a través de iteraciones (De Benito \& Salinas, 2016). En las iteraciones (1) se analiza qué necesidades de mejora se plantean en experiencias MOOC, y qué indicadores están relacionados con estos comportamientos, al punto de poder sugerir relaciones suficientes para implementar cambios en dichos indicadores; posteriormente (2), 
utilizando las analíticas de aprendizaje y herramientas de machine learning, se verifica cuáles de los indicadores encontrados son aptos como predictores de las problemáticas al aplicarlos en el contexto de la UPV, y (3) se proponen procedimientos automáticos para implementar propuestas de mejora que permitan utilizar esos indicadores validados para mejorar la experiencia de los cursos y solventar las problemáticas detectadas.

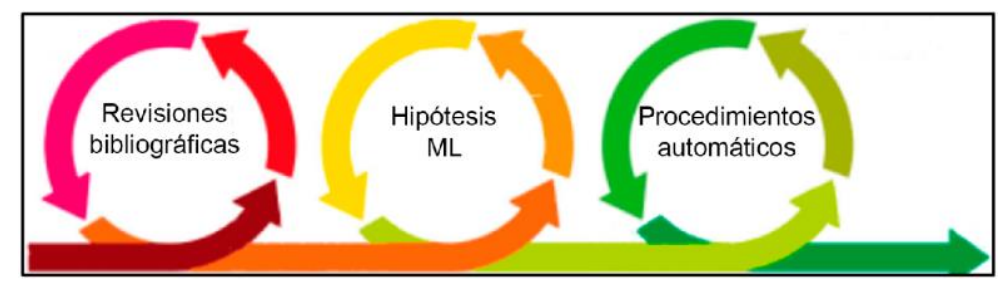

Figura 1. Realimentación de las tres iteraciones que tiene la investigación

\section{MÉTODO}

En esta investigación se trabaja con un gran conjunto de datos en los que se reflejan todas las acciones que realizan 700.000 participantes en 260 cursos en la plataforma MOOC de la UPV desde el año 2015 hasta 2019.

Como se ha comentado, para la resolución del problema se realiza una IBD basada en tres iteraciones, de modo que a continuación en la Tabla 1, se desglosan las preguntas de investigación que se deben resolver en cada iteración.

Tabla 1. Preguntas de investigación en cada iteración.

\begin{tabular}{|c|c|}
\hline Iteración & Preguntas \\
\hline \multirow[t]{3}{*}{$\begin{array}{c}\text { Iteración } \\
\text { I }\end{array}$} & $\begin{array}{l}\text { ¿Cuáles con las características de los MOOC que pueden implicar mejoras a los } \\
\text { participantes, a los cursos y a la Universidad? }\end{array}$ \\
\hline & $\begin{array}{l}\text { ¿Cuáles son las variables más utilizadas para hacer proyecciones de actuación de los } \\
\text { participantes en los cursos MOOC que sirvan para mejorar los procesos y lograr la } \\
\text { calidad de los cursos? }\end{array}$ \\
\hline & $\begin{array}{l}\text { ¿Qué otras variables nos sugieren la Educación a distancia para mejorar la eficacia de } \\
\text { los cursos? }\end{array}$ \\
\hline \multirow[t]{3}{*}{$\begin{array}{l}\text { Iteración } \\
\text { II }\end{array}$} & $\begin{array}{l}\text { ¿De los indicadores obtenidos en la fase } 1 \text {, cuáles se pueden aplicar a los datos } \\
\text { aportados por la UPV? }\end{array}$ \\
\hline & $\begin{array}{l}\text { ¿Qué tipo de tratamiento y filtrado de datos es necesario para obtener los indicadores } \\
\text { de abandono que se han seleccionado? }\end{array}$ \\
\hline & $\begin{array}{l}\text { ¿Cuáles de los indicadores seleccionados realmente funcionan para relacionar el } \\
\text { abandono en los cursos de la UPV? }\end{array}$ \\
\hline \multirow[t]{3}{*}{$\begin{array}{l}\text { Iteración } \\
\text { III }\end{array}$} & $\begin{array}{l}\text { ¿Qué tipo de procedimientos automatizados se plantean para poner en } \\
\text { funcionamiento los indicadores de abandono seleccionados? }\end{array}$ \\
\hline & $\begin{array}{l}\text { ¿Está la plataforma de MOOC de la UPV preparada para poner en funcionamiento los } \\
\text { procedimientos propuestos? }\end{array}$ \\
\hline & $\begin{array}{l}\text { ¿Cuál de las propuestas son de aplicación directa en la plataforma y cuáles no se } \\
\text { pueden o no se desean aplicar? }\end{array}$ \\
\hline
\end{tabular}


En la primera iteración de este trabajo se realizó el análisis y definición del problema través de un procedimiento de revisiones sistemáticas sucesivas de literatura. En esta primera iteración (Martínez-Navarro, 2021) se aborda el abandono en los MOOC desde una perspectiva pedagógica que nos permita conocer los indicadores de abandono detectados en la literatura científica sobre MOOC. En la Figura 2 se muestra un resumen de la metodología utilizada en esta iteración.

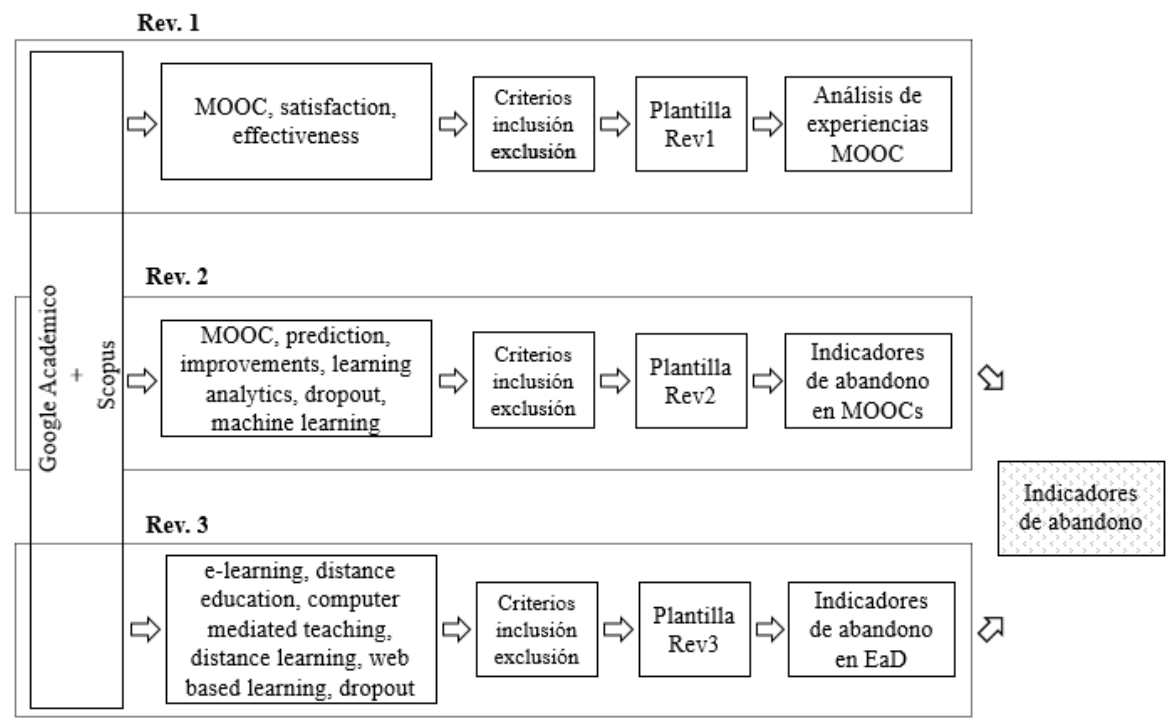

Figura 2. Resumen metodología de la Iteración I (Martínez-Navarro, 2021).

La segunda iteración se centró en verificar si los indicadores de abandono seleccionados en la iteración anterior sirven como predictores con los datos disponibles de la plataforma de la UPV; obteniendo como resultado, un listado con los indicadores que si se consideran aptos para tal tarea. Como primera etapa de esta iteración, es necesario conocer si cada indicador seleccionado tiene su reflejo (directo o a través de operaciones) en el conjunto de datos disponibles, para descartar aquellos de los que no se dispongan datos. Con el listado de "indicadores con datos", se desarrollan un conjunto de scripts encargados del filtrado, procesamiento y obtención de los valores de esos indicadores para cada curso y participante. Posteriormente, se plantea el problema como un contraste de hipótesis en el que con cada uno de los "indicadores con datos" se traza una hipótesis que hay que validar o rechazar en caso de que sea capaz o no de predecir la problemática en los MOOC de la UPV. Dado el gran número de datos de los que se dispone y la necesidad de buscar patrones entre ellos, para verificar las hipótesis se planteará un problema de aprendizaje supervisado mediante Machine Learning del que se disponen pares ordenados variable_hipótesis/problemática.

Se define previamente qué clasificador o algoritmo se utilizará, de modo que, si para una hipótesis se consigue clasificar la mayoría de los datos correctamente, se puede asumir que actúa también de forma precisa con nuevos datos y, por tanto, la hipótesis es acertada. Si, por el contrario, comete muchos errores, se admite que es un modelo erróneo, descartando la hipótesis. Se realizan pruebas con un curso piloto para seleccionar tanto el clasificador óptimo como las métricas y umbrales que se van a tomar como referencia para la validación. Para la ejecución del algoritmo se utilizará como conjunto de entrenamiento el $20 \%$ de los datos disponibles. Una vez finalizado el procedimiento, los datos resultantes serán presentados a los expertos de la UPV para su discusión y validación en caso de que proceda. Por tanto, el resultado de esta segunda iteración será un listado con las hipótesis que han sido validadas con ML en el contexto de la UPV. A continuación, en la Figura 3 se muestra un resumen de la metodología a seguir en la segunda iteración. 


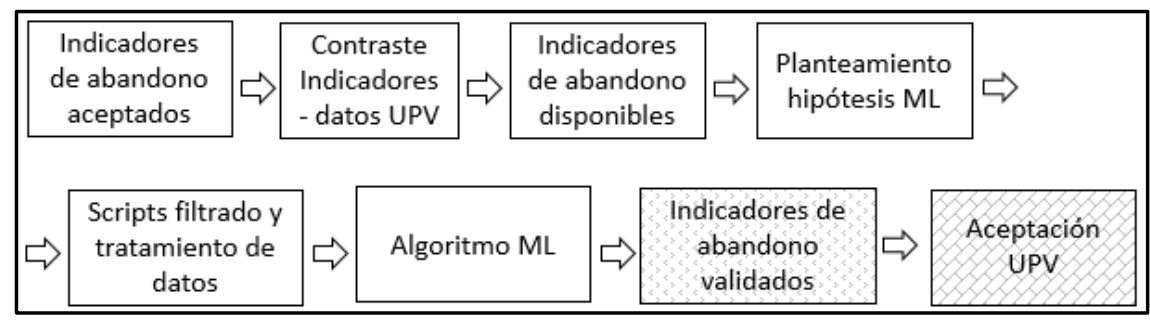

Figura 3. Resumen metodología de la Iteración II.

Independientemente de la validez de las hipótesis/indicadores, la necesidad de la UPV -y el foco principal de este estudio- es diseñar un conjunto de mecanismos automatizados que, basados en la explotación de la gran cantidad de datos disponibles a través de las analíticas de los cursos, puedan potencialmente mejorar la experiencia de los usuarios y de la propia universidad.

Dado que los datos con los que se trabaja están almacenados en grandes bases de datos (BBDD), es obvio que las medidas automatizadas que se propongan deben nacer también de esas BBDD. En concreto, las propuestas a implementar consisten en un conjunto de triggers en los que cada uno tiene como llamada de activación uno de los indicadores validados con las hipótesis, y como acción a ejecutar una comunicación al alumnado y/o docentes con la incidencia detectada. Como vía de comunicación se escoge el correo electrónico al no depender de la propia plataforma MOOC y permitir enviar mensajes de cualquier tamaño. Por otro lado, debido a que los indicadores pueden ser de diversa índole, se considera acertado que los propios docentes pudieran recibir también notificaciones al respecto contemplando, por tanto, mecanismos tanto para estudiantes como para docentes.

\section{RESULTADOS}

Una vez que se han seleccionado los indicadores de abandono en la iteración I que se resumen en la Tabla 2, y como se ha descrito en el apartado de metodología, es necesario comprobar de cuál de estos indicadores se disponen de datos en la plataforma MOOC de la UPV.

Tabla 2. Indicadores de Abandono seleccionados en la Iteración I. (Martinez-Navarro, 2020)

\begin{tabular}{ll}
\hline \multicolumn{1}{c}{ Indicador } & \multicolumn{1}{c}{ Autor } \\
\hline Cantidad de veces que se interactúa en el foro . & Kizilcec et al. (2017) \\
Visualizaciones de un mismo vídeo. & Kizilcec et al. (2017) \\
Contacto/intercambio alumnado - profesor. & Millecamp et al. (2018), Billings et al. \\
& (2001), Chickering \& Gamson (1987) \\
Interacciones en las tareas y recursos. & García-Tinizaray et al. (2015) \\
$\begin{array}{l}\text { Variables de tipo demográfico y profesional. } \\
\text { Ausencia del curso en un periodo superior a un mes. }\end{array}$ & León-Urrutia et al. (2017) \\
Visualización de menos del 50\% de los vídeos en el curso. & Halawa et al. (2014) \\
$\begin{array}{l}\text { Inicio del curso después de dos semanas. } \\
\text { Omisión de vídeos en la unidad anterior. }\end{array}$ & Halawa et al. (2014) \\
$\begin{array}{l}\text { Puntuaciones bajas en las pruebas en las tres primeras } \\
\text { semanas. }\end{array}$ & Halawa et al. (2014) \\
$\begin{array}{l}\text { Intentos para resolver las pruebas en la primera semana. } \\
\text { Interés del estudiante por la materia. }\end{array}$ & Mi \& Yeung (2015) \\
$\begin{array}{l}\text { Abandono si hay ausencias de diez días o más. } \\
\text { Mentalidad, autoeficacia, establecimiento de objetivos y } \\
\text { pertenencia social. }\end{array}$ & Halawa et al. (2014) \\
\hline
\end{tabular}




\begin{tabular}{ll}
\hline \multicolumn{1}{c}{ Indicador } & \multicolumn{1}{c}{ Autor } \\
\hline Interacciones en los foros. & Li et al. (2016) \\
$\begin{array}{l}\text { Navegación (clics) que se realiza en la plataforma. } \\
\text { № de visualizaciones y participaciones en el foro. }\end{array}$ & Kloft et al. (2014) \\
$\begin{array}{l}\text { Participaciones en las videoconferencias, interacción, } \\
\text { visualizaciones y toma de anotaciones. }\end{array}$ & Sinha et al. (2014) \\
$\begin{array}{l}\text { Mensajes y acceso a los foros. } \\
\begin{array}{l}\text { Abandono si no hay ninguna actividad en diez días. } \\
\text { Encuesta inicial de motivación. }\end{array}\end{array}$ & $\begin{array}{l}\text { Rosé et al. (2014), García-Tinizaray et } \\
\text { ldentificación institucional, compromiso académico e } \\
\text { identificación profesional. }\end{array}$ \\
$\begin{array}{l}\text { Experiencia académica previa e influencia de los aspectos } \\
\text { psicológicos, el rendimiento escolar y las metas educativas. }\end{array}$ & Henderikx et al. (2017) \\
$\begin{array}{l}\text { Autorregulación para atender tanto el trabajo académico } \\
\text { como sus compromisos sociales y laborales. }\end{array}$ & Tinto (1999) \\
$\begin{array}{l}\text { Edad y sexo (biodatos). } \\
\text { Kember (1989), Escanés et al. (2014) }\end{array}$ & \\
\hline
\end{tabular}

Analizando estos datos, dado que la información disponible sobre las interacciones en el foro es enorme, se decide desechar los indicadores de abandono que están relacionados con el foro, ya que es necesario una investigación personalizada solo para este apartado y, por las necesidades de la propia institución, se ha decidido abordar este análisis en un trabajo posterior. Con esas indicaciones, el listado de los indicadores de abandono con los que trabajar se reduce a tan solo diez, según se describe en la Tabla 3.

Tabla 3. Indicadores de abandono con datos disponibles de la plataforma de la UPV

\begin{tabular}{cc}
\hline $\begin{array}{c}\text { Variables de tipo demográfico y profesional } \\
\text { Ausencia en un periodo superior a } 10 \text { días }\end{array}$ & $\begin{array}{c}\text { Inicio del curso después de } 2 \text { semanas } \\
\text { Visualizaciones de un mismo vídeo. } 50 \% \text { de visualizaciones de los vídeos } \\
\text { en las primeras semanas }\end{array}$ \\
$\begin{array}{c}\text { Ausencia en un periodo superior a } 1 \text { mes } \\
\text { Omisión de vídeos de la unidad anterior }\end{array}$ & $\begin{array}{c}\text { Abandono si hay ausencias de diez días o más. } \\
\text { semana }\end{array}$ \\
& $\begin{array}{c}\text { Puntuaciones bajas en las pruebas en las tres } \\
\text { primeras semanas. }\end{array}$
\end{tabular}

Con los indicadores seleccionados se trazan hipótesis que hay que validar para concretar en qué casos se considera que son capaces de predecir - a nivel de análisis de datos- abandonos en los MOOC de la UPV.

Para ello, se parte de los diez identificadores seleccionados (Tabla 3), utilizando como hipótesis demográfica y profesional "Edad y género", que se utiliza con un duplicado "Edad, género y nivel educativo". Además, dada la importancia de esta última, y considerándola un complemento ideal para las otras hipótesis planteadas, se decide añadir "Edad, género y nivel educativo" a las otras hipótesis desdoblándolas con la identificación "bis".

En total, como se muestra en la Tabla 4, se plantean veinte hipótesis distintas.

Tabla 4. Hipótesis generadas para verificar

\begin{tabular}{llll}
\hline 1 & Edad y género & & \\
2 & Edad, género y nivel educativo & & \\
3 & Omisión de tareas en 10 días & 3 bis & + Edad, género y nivel educativo \\
4 & Tiempo dedicado a ver vídeos & 4 bis & + Edad, género y nivel educativo \\
5 & Ausencia en un periodo superior a un mes & 5 bis & + Edad, género y nivel educativo \\
\hline
\end{tabular}




\begin{tabular}{llll}
\hline 6 & Ausencia en un periodo de 10 días & 6 bis & + Edad, género y nivel educativo \\
7 & $\begin{array}{l}\text { Visualización de vídeos inferior al } 50 \% \text { en las } \\
\text { tres primeras semanas }\end{array}$ & 7 bis & + Edad, género y nivel educativo \\
8 & $\begin{array}{l}\text { Inicio del curso dos semanas después del } \\
\text { registro }\end{array}$ & 8 bis & + Edad, género y nivel educativo \\
9 & $\begin{array}{l}\text { Omisión de vídeos de la unidad anterior } \\
10\end{array}$ & $\begin{array}{l}\text { Puntuaciones bajas en las pruebas en las tres } \\
\text { primeras semanas }\end{array}$ & + Edad, género y nivel educativo \\
11 & $\begin{array}{l}\text { Intentos para resolver las pruebas en la } \\
\text { primera semana. }\end{array}$ & + Edad, género y nivel educativo \\
\end{tabular}

Con la ejecución de los scripts de filtrado se genera el fichero necesario con solo la información necesaria para validar cada una de las hipótesis planteadas. Siendo este proceso necesario realizarlo para los 260 cursos de los que se dispone información.

El siguiente paso fue comprobar si estas hipótesis están relacionadas con el abandono de los estudiantes. Para ello nos enfrentamos ante un problema de aprendizaje supervisado del cual hay que elegir su clasificador a partir de un conjunto de pruebas que se realizan con los datos de un curso piloto, al que se aplicarán los seis modelos de Machine Learning más utilizados (Russo et al, 2016).

A continuación, en la Tabla 5 se muestran los resultados obtenidos por cada modelo de ML; observando que el algoritmo que mejor puntuación obtiene, y por tanto el elegido, es Ramdon Forest.

Tabla 5. Comparativa de resultados con diferentes algoritmos Machine Learning

\begin{tabular}{lccccc}
\hline \multicolumn{1}{c}{ Precisión (\%) } & \multicolumn{5}{c}{ Hipótesis iniciales en el curso de prueba } \\
& 1 & 2 & 3 & 4 & 5 \\
\hline Logistic Regression & 93,432 & 98,952 & 98,802 & 93,432 & 93,432 \\
Decision Tree classification & 93,282 & 99,230 & 99,080 & 93,432 & 93,132 \\
KNeighbors classification & 93,325 & 98,930 & 98,695 & 93,432 & 93.261 \\
Support vector machine & 93,432 & 98,802 & 98,930 & 93,432 & 94,432 \\
Naive Bayes & 93,432 & 98,952 & 97,411 & 93,432 & 94,432 \\
Random Forest & 93,389 & 99,358 & 99,337 & 93,432 & 94,432 \\
\hline
\end{tabular}

Con los datos que aporta la UPV, se observa que el número de abandonos en los MOOC de la UPV es muy alto, entorno al $80 \%$, lo que indica que el tipo de problema a resolver tiene una de sus clases (la del abandono) con un gran peso frente a la del no abandono. O lo que es lo mismo, cualquier algoritmo que siempre diera por resultado "Abandono", tendría un $80 \%$ de precisión, que es un valor muy alto. Esto da lugar a una matriz de confusión que describe el rendimiento del modelo similar al que se muestra en la Figura 4.

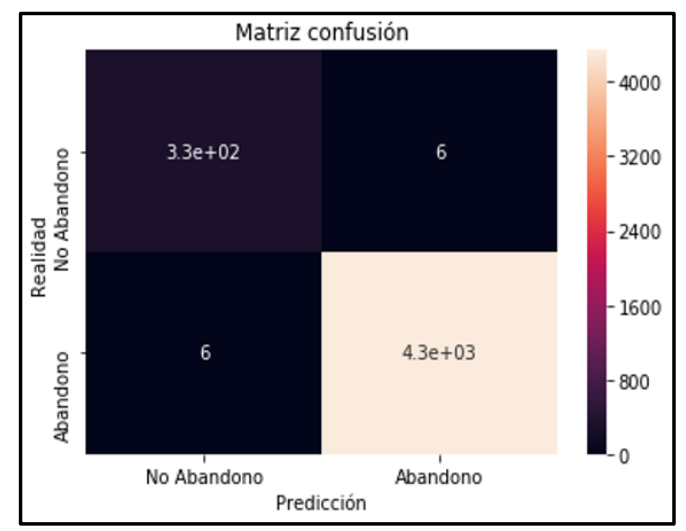

Figura 4. Matriz de confusión del problema Machine Learning 
Dado el tipo de problema a resolver, y con la finalidad de reducir los falsos negativos y falsos positivos de las posibles métricas a elegir que ofrece el algoritmo, se decide seleccionar tres:

- Precisión: número de elementos identificados correctamente como positivos de un total de elementos identificados como positivos.

- Recall: número de elementos identificados correctamente como positivos del total de positivos verdaderos.

- F1-score: media armónica que combina los valores de la precisión y recall.

Seleccionado el algoritmo y las métricas, se desarrolla un nuevo script que implementa la resolución del problema utilizando como conjunto de pruebas del $20 \%$ de los datos disponibles. Este script deberá calcular las métricas para las 20 hipótesis planteadas y para los 260 cursos de los que se disponen datos, obteniendo los resultados que se muestran en la Tabla 6 para cada curso:

Tabla 6. Resultados con técnicas de ML para el curso Reacciones químicas y cálculos estequiom. 2016.

\begin{tabular}{|c|c|c|c|c|c|c|c|c|c|c|c|}
\hline Hipótesis & 1 & 2 & 3 & 4 & 5 & 6 & 7 & 8 & 9 & 10 & 11 \\
\hline Precisión & 0,45 & 0,48 & 0,93 & 0,80 & 0,83 & 0,90 & 0,90 & 0,45 & 0,94 & 0,96 & 0,91 \\
\hline Recall & 0,45 & 0,46 & 0,98 & 0,85 & 0,90 & 0,97 & 0,83 & 0,45 & 0,84 & 0,92 & 0,95 \\
\hline F1-score & 0,45 & 0,48 & 0,90 & 0,77 & 0,94 & 0,89 & 0,84 & 0,51 & 0,96 & 0,87 & 0,96 \\
\hline Hipótesis & & & 3bis & 4bis & 5bis & 6bis & 7bis & 8bis & 9bis & 10bis & 11bis \\
\hline Precisión & & & 0,92 & 0,89 & 0,83 & 0,94 & 0,78 & 0,46 & 0,85 & 0,90 & 0,91 \\
\hline Recall & & & 0,90 & 0,77 & 0,99 & 0,95 & 0,86 & 0,50 & 0,81 & 0,94 & 0,94 \\
\hline F1-score & & & 0,94 & 0,82 & 0,94 & 0,94 & 0,84 & 0,45 & 0,83 & 0,88 & 0,94 \\
\hline
\end{tabular}

Con estas métricas de clasificación obtenidas para cada curso, es necesario marcar un umbral de decisión que determine la validación de las hipótesis. Nuevamente a partir del curso piloto, se decide como umbral apropiado valores superiores al $80 \%$; por lo que, aplicando este umbral a cada curso, conseguimos el porcentaje de cursos que han superado el mismo para cada métrica en una única tabla (Tabla 7):

Tabla 7. Porcentaje de cursos por encima del umbral establecido.

\begin{tabular}{|c|c|c|c|c|c|c|c|c|c|c|c|}
\hline Hipótesis & 1 & 2 & 3 & 4 & 5 & 6 & 7 & 8 & 9 & 10 & 11 \\
\hline Precisión & 0 & 0 & 100 & 75 & 100 & 100 & 68 & 0 & 100 & 100 & 100 \\
\hline Recall & 0 & 0 & 100 & 51 & 100 & 100 & 48 & 0 & 93 & 100 & 100 \\
\hline F1-score & 0 & 0 & 100 & 61 & 100 & 100 & 60 & 0 & 100 & 100 & 100 \\
\hline Hipótesis & & & 3bis & 4bis & 5bis & 6bis & 7bis & 8bis & 9bis & 10bis & 11bis \\
\hline Precisión & & & 100 & 95 & 98 & 100 & 87 & 0 & 100 & 100 & 100 \\
\hline Recall & & & 100 & 50 & 100 & 100 & 61 & 0 & 92 & 100 & 100 \\
\hline F1-score & & & 100 & 72 & 100 & 100 & 67 & 0 & 100 & 100 & 100 \\
\hline
\end{tabular}

Del mismo modo, se marca un segundo umbral para terminar de clasificar las hipótesis. Se validarán las hipótesis en caso de que algunas de sus métricas sean superiores a un $85 \%$ : se considera más importante una posible detección de abandono (aunque esta resulte finalmente falsa) que una no detección de ese abandono por la no inclusión de una hipótesis. Con la aplicación de este segundo umbral, finalmente ya disponemos de las hipótesis validadas y que servirán como base de nuestra propuesta de mejora de experiencia.

En concreto son las que se muestran en la Tabla 8. 
Tabla 8. Listado de hipótesis validadas.

\begin{tabular}{|c|c|}
\hline Hipótesis & Descripción \\
\hline 3 y 3bis & $\begin{array}{l}\text { El alumnado no realiza las tareas asignadas en un periodo de diez días; también se } \\
\text { tendrán en cuenta la edad, género y nivel educativo (bis). }\end{array}$ \\
\hline 4 bis & $\begin{array}{l}\text { El estudiante dedica un tiempo determinado a visualizar los vídeos de cada semana, } \\
\text { teniendo en cuenta la edad, género y nivel educativo. }\end{array}$ \\
\hline 5 y 5 bis & $\begin{array}{l}\text { El estudiante tiene una ausencia durante un periodo superior a un mes desde su última } \\
\text { conexión con la plataforma; también se tendrán en cuenta la edad, género y nivel } \\
\text { educativo (bis). }\end{array}$ \\
\hline 6 y 6 bis & $\begin{array}{l}\text { El estudiante tiene una ausencia durante un periodo de diez días desde su última } \\
\text { conexión con la plataforma; también se tendrán en cuenta la edad, género y nivel } \\
\text { educativo (bis). }\end{array}$ \\
\hline 7 bis & $\begin{array}{l}\text { Durante las tres primeras semanas, el estudiante visualiza menos del } 50 \% \text { de los } \\
\text { vídeos asignados. }\end{array}$ \\
\hline 9 y 9 bis & $\begin{array}{l}\text { El estudiante no ha visualizado todos los vídeos de la unidad anterior a la que se está } \\
\text { cursando; también se tendrán en cuenta la edad, género y nivel educativo (bis). }\end{array}$ \\
\hline 10 y 10 bis & $\begin{array}{l}\text { El estudiante ha obtenido puntuaciones bajas en las pruebas realizadas durante las } \\
\text { primeras tres semanas del curso; también se tendrán en cuenta la edad, género y nivel } \\
\text { educativo (bis). }\end{array}$ \\
\hline 11 y 11 bis & $\begin{array}{l}\text { El estudiante necesita de varios intentos para resolver las pruebas de la primera } \\
\text { semana del curso; también se tendrán en cuenta la edad, género y nivel educativo } \\
\text { (bis). }\end{array}$ \\
\hline
\end{tabular}

La tercera y última de las iteraciones consiste en plantear un conjunto de propuestas que, partiendo de las hipótesis validadas, den solución al objetivo principal que es la mejora de la experiencia de los MOOC y más concretamente la reducción del porcentaje de abandonos. Para este propósito, se plantea el mecanismo automatizado de envío de notificaciones personalizadas al alumnado y docentes en caso de que algunas de las hipótesis validadas se activen, para intentar volver a atraer al alumnado al curso.

A continuación, se desglosan en la Tabla 9 cada uno de los mensajes predefinidos a enviar en caso de que se active alguno de los indicadores:

Tabla 9. Relación hipótesis - propuesta automática de mejora. Elaboración propia

\begin{tabular}{cccc}
\hline & Hipótesis & \multicolumn{2}{c}{ Email automático } \\
profesores
\end{tabular}


Finalizada la última iteración, en una etapa de reflexión, se realiza una presentación formal de los mecanismos propuestos ante los responsables de los MOOC en la UPV, previo envío de un informe ejecutivo de la investigación.

La primera aportación de los expertos es que, a día de hoy, la UPV todavía no dispone de un módulo de envío automatizado de correos electrónicos personalizado, está en desarrollo y por tanto, hay que esperar a que esté disponible para implementar las propuestas de mejoras seleccionadas.

Posteriormente se realiza un análisis pormenorizado de cada una de las propuestas realizadas. En concreto, la hipótesis "4bis" (tiempo dedicado a ver vídeos) es rechazada ya que, como indican, es necesario tener modelizado previamente para cada curso el tiempo de dedicación objetivo, y esa información no se dispone en los nuevos cursos. Además, se indica que por su experiencia y dada la longitud de sus MOOC, todo estudiante que tiene un periodo de cuatro semanas sin interactuar lo dan por abandonado, de modo que no es necesario enviar ningún tipo de notificación; quedando las propuestas 5 y " 5 bis" descartadas.

Con estas reflexiones, los mecanismos automáticos para la mejora de la tasa de abandono que quedan aprobados son los que se muestran en la Tabla 10.

Tabla 10. Mecanismos automáticos para la mejora de la tasa de abandono

\begin{tabular}{ccc}
\hline Hipótesis & Email automático estudiantes & Email automático para profesores \\
\hline 3 y 3bis & $\begin{array}{c}\text { Estudiante: tareas que no ha realizado } \\
\text { hasta la fecha. }\end{array}$ & \\
7 bis & $\begin{array}{c}\text { Estudiante: recordatorio de la } \\
\text { importancia de ver los vídeos asignados. } \\
9 \text { y 9bis }\end{array}$ & $\begin{array}{c}\text { Estudiante: contenidos de los vídeos } \\
\text { que no ha visualizado. }\end{array}$ \\
10 y $10 \mathrm{bis}$ & $\begin{array}{c}\text { Estudiante: motivación para el alumno. } \\
\text { su identificador. }\end{array}$ & \\
11 y 11 bis & Estudiante: motivación para el alumno. & \\
\hline
\end{tabular}

\section{DISCUSIÓN Y CONCLUSIONES}

Al comenzar este trabajo se planteaban proponer mecanismos automatizados que se pudieran poner en marcha dentro de los MOOC de la UPV. Pero es importante destacar, que esta investigación ha seguido siempre criterios pedagógicos y basados en las revisiones bibliográficas realizadas para el avance en cada una de las iteraciones, alejándose de la perspectiva simple de minería de datos.

Se abordaron objetivos específicos y preguntas de investigación que se pretendían ir cubriendo en cada una de las etapas y que creemos que se han cumplido así:

Desarrollar modelos predictivos, basados en la analítica de aprendizaje, que describan el posible comportamiento de los participantes de los cursos MOOC: el objetivo se ha conseguido gracias al amplio trabajo de procesamiento y filtrado de los datos proporcionados por la UPV y las técnicas de ML utilizadas. Con los indicadores de abandono seleccionados a partir del listado obtenido en la Iteración I, se han podido formular y validar un conjunto de hipótesis de las cuales se han logrado métricas esperanzadoras para la predicción de patrones de comportamiento en los abandonos.

En estos últimos dos años se siguen desarrollando nuevos modelos predictivos en entornos MOOC como el de Hmedna et al. (2020) cuyo objetivo es predecir los estilos de aprendizaje del alumnado MOOC en función de sus huellas de aprendizaje a partir del análisis de los datos recopilados en un curso de la misma plataforma edX; o como el modelo desarrollado por Jin (2020) en el que, también con el objetivo de reducir la deserción, se toman como parámetros los datos del comportamiento de aprendizaje, los hábitos de estudio y el tiempo de aprendizaje de los diferentes estudiantes en MOOC.

Proponer estrategias basadas en analíticas de aprendizaje, para mejorar la experiencia de los estudiantes y de la universidad y los elementos didácticos de los MOOC de la UPV: El objetivo 
principal no trataba sobre indicadores, sino sobre estrategias y procedimientos automatizados. Se ha cumplido al plantear la dupla trigger + email, en el que, tras la activación de un indicador de abandono, se envía un email al usuario afectado. Esta estrategia, al igual que el resto de los elementos obtenidos en cada una de las iteraciones de la investigación, fue validada por los expertos de la UPV.

En investigaciones actuales se continúa analizando el uso de otras estrategias para reducir la tasa de abandono. Así, por ejemplo, en Ortega-Arranz et al. (2019), se analizan las estrategias de gamificación basadas en recompensas para promover la participación de los estudiantes y prevenir la deserción, a pesar de que no tuvieron un efecto significativo en la retención y el compromiso de los estudiantes.

Proponer procedimientos automatizados para poner en funcionamiento los indicadores de abandono seleccionados: finalmente, se han validado un total de once procedimientos automatizados; no obstante, algunos de los procedimientos propuestos no se pueden aplicar en este momento debido a que la UPV no cuenta actualmente con la infraestructura necesaria para ello.

En investigaciones recientes como la de Borrella et al. (2019) se proponen mecanismos similares a los diseñados: en esta, con la aplicación de algoritmos de aprendizaje automático sobre datos de flujo de clics, se pretenden identificar al alumnado en riesgo de abandonar y se diseña una intervención que consiste en enviar correos electrónicos de ánimo personalizados a los estudiantes en riesgo.

Una de las conclusiones no esperadas durante la investigación es que, de los veinticinco indicadores pedagógicos de abandono referidos por las revisiones bibliográficas, solo se validan diez de ellos en los cursos de la UPV (no se tienen datos automáticos ni automatizables de los otros), y finalmente, solo seis de ellos son posibles predictores del abandono de alumnado. A pesar de la ingente cantidad de datos de los que se disponen, se echa en falta información sobre otras variables, como el estado de ánimo del alumnado, datos de los docentes o si se complementa la plataforma MOOC con otros medios o redes sociales. Aunque con learning analytics y machine learning se obtiene el máximo rendimiento de los datos disponibles, no siempre funcionan correctamente al no poder tener disponibles todos los datos deseables, lo cual pone de relieve la importancia de una estrategia de implementación de analíticas prospectiva y no solo reactiva por parte de las instituciones, de manera que no solo se trabaje con las analíticas proporcionadas por las plataformas, sino que se avance hacia modelos en los que las analíticas - o los resultados provistos por ellas- sean codiseñadas por los usuarios; además de hacer énfasis en la necesidad de que el uso estas analíticas sea promovido por la institución como parte fundamental del diseño educativo.

\section{REFERENCIAS BIBLIOGRÁFICAS}

Bartolomé-Pina, A., \& Steffens, K. (2015) ¿Son los MOOC una alternativa de aprendizaje? Comunicar, 22(44), 91-99. http://dx.doi.org/10.3916/C44-2015-10

Bean, J., \& Metzner, B. (1985). A Conceptual Model of Nontraditional Undergraduate Student Attrition. Review of Educational Research, 55(4), 485-540. https://doi.org/10.3102/00346543055004485

Billings, D. M., Connors, H. R., \& Skiba, D. J. (2001). Benchmarking best practices in Webbased nursing courses. Advances in Nursing Science, 23, 41-52. https://doi.org/10.1097/00012272$\underline{200103000-00005}$

Bishop C.M. (2006) Pattern recognition and machine learning. Springer-Verlag.

Borrella, I., Caballero-Caballero, S., \& Ponce-Cueto, E. (2019). Predict and Intervene: Addressing the Dropout Problem in a MOOC-based Program. In Proceedings of the Sixth (2019) ACM Conference on Learning@ Scale, 1-9. https://doi.org/10.1145/3330430.3333634

Chickering, A. W., \& Gamson, Z. F. (1987). Seven principles for good practice in undergraduate education. AAHE bulletin, 3(7). https://doi.org/10.1080/13562517.2013.827653 
De Benito, B., \& Salinas, J. (2016). La Investigación Basada en Diseño en Tecnología Educativa. Revista Interuniversitaria de Investigación en Tecnología Educativa, 0, 44-59. https://doi.org/10.6018/riite2016/260631

Escanés, G., Herrero, V., Merlino, A., \& Ayllón, S. (2014). Deserción en educación a distancia: factores asociados a la elección de modalidad como desencadenantes del abandono universitario. Virtualidad, Educación Y Ciencia, 5(9), 45-55. https://bit.ly/31UD380

García-Tinizaray, D., Ordoñez-Briceño, K., \& Torres-Diaz, J. C. (2015). Learning analytics para predecir la deserción de estudiantes a distancia. Campus virtuales, 3(1), 120-126. https://bit.ly/3wA06my

Halawa, S., Greene, D., \& Mitchell, J. (2014). Dropout prediction in MOOC using learner activity features. Proceedings of the Second European MOOC Stakeholder Summit, Lausanne, Switzerland, 58-65.

Henderikx, M. A., Kreijns, K., \& Kalz, M. (2017). Refining success and dropout in massive open online courses based on the intention-behavior gap. Distance Education, 38(3), 353-368. https://doi.org/10.1080/01587919.2017.1369006

Hmedna, B., El Mezouary, A., \& Baz, O. (2020). A predictive model for the identification of learning styles in MOOC environments. Cluster Comput, 23, 1303-1328. https://doi.org/10.1007/s10586-019-02992-4

Jin, C. (2020). MOOC student dropout prediction model based on learning behavior features and parameter optimization. Interactive Learning Environments. https://doi.org/10.1080/10494820.2020.1802300

Kember, D. (1989). A longitudinal-process model of drop-out from distance education. The Journal of Higher Education, 60(3), 278-301. https://doi.org/10.1080/00221546.1989.11775036

Kizilcec, R. F., Pérez-Sanagustín, M., \& Maldonado, J. J. (2017). Self-regulated learning strategies predict learner behavior and goal attainment in Massive Open Online Courses. Computers \& education, 104, 18-33. https://doi.org/10.1016/i.compedu.2016.10.001

Kloft, M., Stiehler, F., Zheng, Z., \& Pinkwart, N. (2014). Predicting MOOC dropout over weeks using machine learning methods. Proceedings of the EMNLP 2014 Workshop on Analysis of Large Scale Social Interaction in MOOC, 60-65. https://doi.org/10.3115/v1/w14-4111

Lee, Y., \& Choi, J. (2011). A Review of Online Course Dropout Research: Implications for Practice and Future Research. Educational Technology Research and Development, 59(5), 593-618. https://doi.org/10.1007/s11423-010-9177-y

León-Urrutia, M., Vázquez-Cano, E., \& López-Meneses, E. (2017). MOOC learning analytics using real-time dynamic metrics. @tic. Revista d'innovació educativa, 18(3). https://doi.org/10.7203/attic.18.10022

Li, W., Gao, M., Li, H., Xiong, Q., Wen, J., \& Wu, Z. (2016). Dropout prediction inMOOCusing behavior features and multi-view semi-supervised learning. Proceedings of the 2016 International Joint Conference on Neural Networks (IJCNN), 3130-3137. https://doi.org/10.1109/IJCNN.2016.7727598

Liang, J., Yang, J., Wu, Y., Li, C., \& Zheng, L. (2016). Big data application in education: dropout prediction in edx MOOC. Proceedings of the IEEE Second International Conference on Multimedia Big Data (BigMM), 440-443, https://doi.org/10.1109/BigMM.2016.70

Martinez-Navarro, J. A. (2021). Indicadores de abandono en contextos MOOC, una aproximación pedagógica desde la literatura. Universitas Tarraconensis. Revista de Ciències de l'Educació, 3, 36-59. https://doi.org/10.17345/ute.2020.3.3031 
Mi, F., \& Yeung, D. Y. (2015). Temporal Models for Predicting Student Dropout in Massive Open Online Courses. 2015 IEEE International Conference on Data Mining Workshop. https://doi.org/10.1109/icdmw.2015.174

Millecamp, M., Gutiérrez, F., Charleer, S., Verbert, K., \& De Laet, T. (2018). A qualitative evaluation of a learning dashboard to support advisor-student dialogues. Proceedings of the 8th International Conference on Learning Analytics and Knowledge, 56-60. https://doi.org/10.1145/3170358.3170417

Mohri, M., Rostamizadeh, A., \& Talwalkar, A. (2018) Foundations of machine learning. MIT press, $1(1-3)$.

Ortega-Arranz, A., Bote-Lorenzo, M.L., Asensio-Pérez, J.I., Martínez-Monés, A., GómezSánchez, E., \& Dimitriadis, Y. (2019). To reward and beyond: Analyzing the effect of rewardbased strategies in a MOOC, Computers \& Education, 142, 103639, https://doi.org/10.1016/j.compedu.2019.103639.

Rolfe, V. (2015). A systematic review of the socio-ethical aspects of massive online open courses. European Journal of Open, Distance and e-Learning, 18(1), 52-72. https://bit.ly/31RC2xp

Rosé, C. P., Carlson, R., Yang, D., Wen, M., Resnick, L., Goldman, P., \& Sherer, J. (2014). Social factors that contribute to attrition in MOOC. Proceedings of the first ACM conference on Learning@ scale conference, 197-198. https://doi.org/10.1145/2556325.2567879

Ruipérez-Valiente, J. A. (2020). El Proceso de Implementación de Analíticas de Aprendizaje. RIED. Revista Iberoamericana de Educación a Distancia, 23(2), 88-101 http://dx.doi.org/10.5944/ried.23.1.26283

Russo, C., Ramón, H., Alonso, N., Cicerchia, L., Esnaola, L., \& Tessore, J.P. (2016). Tratamiento masivo de datos utilizando técnicas de machine learning. XVIII Workshop de Investigadores en Ciencias de la Computación WICC 2016. https://bit.ly/31Vub1S

Shah, D. (2016). Monetization over Massiveness: A Review of MOOC Stats and Trends in 2016. Class Central. https://bit.ly/2PzTxjl

Sinha, T., Jermann, P., Li, N., \& Dillenbourg, P. (2014). Your click decides your fate: Inferring information processing and attrition behavior from mooc video clickstream interactions. Proceeding of the 2014 conference on Empirical Methods in Natural Language Processing (EMNLP), 3-14. https://bit.ly/3usQKqQ

Tinto, V. (1999). Taking retention seriously: Rethinking the first year of college. NACADA Journal, 19(2), 5-9. https://doi.org/10.12930/0271-9517-19.2.5

Veletsianos, G., Collier, A., \& Schneider, E. (2015). Digging deeper into learners' experiences in MOOC s: Participation in social networks outside of MOOC s, notetaking and contexts surrounding content consumption. British Journal of Educational Technology, 46(3), 570-587. https://doi.org/10.1111/bjet.12297

Veletsianos, G., \& Shepherdson, P. (2016). A systematic analysis and synthesis of the empirical MOOC literature published in 2013-2015. The International Review of Research in Open and Distributed Learning, 17(2), 198-221. https://doi.org/10.19173/irrodl.v17i2.2448 


\title{
INFORMACIÓN SOBRE LOS AUTORES
}

\author{
Jorge Ángel Martínez Navarro \\ Universidad de Murcia
}

Profesor en la Escuela de Arte y la Escuela Superior de Diseño de Murcia.

Ingeniero de telecomunicaciones por la Universidad Politécnica de Cartagena, MBA por la Escuela Europea de Negocios y Master en Tecnología Educativa por la Universitat Rovira i Virgili. Ha dedicado la primera etapa de su vida laboral a la gestión de proyectos relacionados con las tecnologías de la información e infraestructuras y ahora está centrado en la docencia e investigación.

\section{Ignacio Despujol Zabala}

Universitat Politècnica de València

Coordinador de la iniciativa MOOC de la UPV.

Ingeniero de telecomunicación e Ingeniero aeronáutico por la Universitat Politècnica de València y MBA executive por la Universidad Católica de Valencia. Tiene la apasionante tarea de incorporar la filosofía 2.0 a la UPV y estudiar la utilidad de las nuevas herramientas de Internet para la docencia reglada, especialmente las plataformas de vídeo en red y los MOOC.

También es profesor asociado en la titulación de Grado de Ingeniería Espacial, donde imparte la asignatura ingeniería aeroportuaria a 130 alumnos en modo flipped.

Los textos publicados en esta revista están sujetos a una licencia de Reconocimiento 4.0 España de Creative Commons. Puede copiarlos, distribuirlos, comunicarlos públicamente y hacer obras derivadas siempre que reconozca los créditos de las obras (autoría, nombre de la revista, institución editora) de la manera especificada por los autores o por la revista. La licencia completa se puede consultar en:Licencia Creative Commons Atribución-NoComercial-Compartir por igual 4.0 Internacional.
} 